\title{
The Numerical Solution of Weakly Singular Volterra Integral Equations By Collocation on Graded Meshes
}

\author{
By Hermann Brunner
}

\begin{abstract}
Since the solution of a second-kind Volterra integral equation with weakly singular kernel has, in general, unbounded derivatives at the left endpoint of the interval of integration, its numerical solution by polynomial spline collocation on uniform meshes will lead to poor convergence rates. In this paper we investigate the convergence rates with respect to graded meshes, and we discuss the problem of how to select the quadrature formulas to obtain the fully discretized collocation equation.
\end{abstract}

1. Introduction. In this paper we present an analysis of certain numerical methods for solving the (nonlinear) Volterra integral equation

$$
y(t)=g(t)+\int_{0}^{t}(t-s)^{-\alpha} \cdot k(t, s, y(s)) d s, \quad t \in I:=[0, T], T<\infty,
$$

where $0<\alpha<1$, and where $g$ and $k$ denote given smooth functions. In practical applications one very frequently encounters the linear counterpart of (1.1),

$$
y(t)=g(t)+\int_{0}^{t}(t-s)^{-\alpha} \cdot K(t, s) y(s) d s, \quad t \in I \quad(0<\alpha<1)
$$

in the subsequent analysis we shall, for ease of exposition, usually utilize the linear version of (1.1) to display the principal ideas.

The numerical methods to be analyzed will be collocation methods in the polynomial spline space,

$$
S_{m-1}^{(-1)}\left(Z_{N}\right):=\left\{u:\left.u\right|_{\sigma_{n}}=: u_{n} \in \pi_{m-1}, 0 \leqslant n \leqslant N-1\right\},
$$

associated with a given partition (or: mesh) $\Pi_{N}$ of the interval $I$,

$$
\Pi_{N}: 0=t_{0}^{(N)}<t_{1}^{(N)}<\cdots<t_{N}^{(N)}=T
$$

(the index indicating the dependence of the mesh points on $N$ will, for ease of notation, subsequently be suppressed). Here, $\pi_{m-1}$ denotes, for given $m \geqslant 1$, the space of (real) polynomials of degree not exceeding $m-1$, and we have set $\sigma_{0}:=$ $\left[t_{0}, t_{1}\right], \sigma_{n}:=\left(t_{n}, t_{n+1}\right](1 \leqslant n \leqslant N-1)$; the set $Z_{N}:=\left\{t_{n}: 1 \leqslant n \leqslant N-1\right\}$ (i.e., the interior mesh points) will be referred to as the knots of these polynomial splines. In addition, we define

$$
h:=\max \left\{h_{n}: 0 \leqslant n \leqslant N-1\right\}, \quad h^{\prime}:=\min \left\{h_{n}: 0 \leqslant n \leqslant N-1\right\},
$$

Received February 3, 1984; revised November 6, 1984.

1980 Mathematics Subject Classification. Primary 65R20; Secondary 45D05.

Key words and phrases. Volterra integral equations, weakly singular kernels, polynomial spline collocation, graded meshes. 
where $h_{n}:=t_{n+1}-t_{n}$; the quantity $h$ is often called the diameter of the mesh $\Pi_{N}$. (Note that, according to the above remark on our notation, both $h$ and $h^{\prime}$ will depend on $N$.)

In order to describe these collocation methods we rewrite (1.1), for $t \in \sigma_{\dot{n}}$, in "one-step form",

$$
y(t)=F_{n}(y ; t)+\int_{t_{n}}^{t}(t-s)^{-\alpha} \cdot k(t, s, y(s)) d s,
$$

where

$$
F_{n}(y ; t):=g(t)+\sum_{i=0}^{n-1} \int_{t_{i}}^{t_{i+1}}(t-s)^{-\alpha} \cdot k(t, s, y(s)) d s
$$

$$
(0 \leqslant n \leqslant N-1)
$$

For given parameters $\left\{c_{j}\right\}$ with $0 \leqslant c_{1}<\cdots<c_{m} \leqslant 1$ we introduce the sets

$$
X_{n}:=\left\{t_{n j}:=t_{n}+c_{j} h_{n}: 1 \leqslant j \leqslant m\right\} \quad(0 \leqslant n \leqslant N-1),
$$

and we define

$$
X(N):=\bigcup_{n=0}^{N-1} X_{n}
$$

the set $X(N) \subset I$ will be referred to as the set of collocation points, while the $c_{j}$ 's will be called collocation parameters. A numerical approximation to the exact solution $y$ of (1.1) (or (1.2)) is an element of $S_{m-1}^{(-1)}\left(Z_{N}\right)$ satisfying the given integral equation on $X(N)$; i.e., by (1.5), this approximation $u$ is computed recursively from

$$
\begin{aligned}
u_{n}\left(t_{n j}\right)= & F_{n}\left(u ; t_{n j}\right) \\
& +h_{n}^{1-\alpha} \cdot \int_{0}^{c_{j}}\left(c_{j}-v\right)^{-\alpha} \cdot k\left(t_{n j}, t_{n}+v h_{n}, u_{n}\left(t_{n}+v h_{n}\right)\right) d v
\end{aligned}
$$

$$
(1 \leqslant j \leqslant m)
$$

where

$$
\begin{aligned}
F_{n}\left(u ; t_{n j}\right):=g\left(t_{n j}\right)+\sum_{i=0}^{n-1} \int_{t_{i}}^{t_{i+1}}\left(t_{n j}-s\right)^{-\alpha} \cdot k\left(t_{n j}, s, u_{i}(s)\right) d s & \\
& (0 \leqslant n \leqslant N-1) .
\end{aligned}
$$

It follows by a standard contraction mapping argument that, for any continuous $k(t, s, y)$ with bounded partial derivative $k_{y}(t, s, y)$, and for any mesh $\Pi_{N}$ whose mesh diameter $h$ tends to zero as $N$ tends to infinity, (1.8) will define a unique approximation $u \in S_{m-1}^{(-1)}(Z)$ for all sufficiently large $N$; once the values $\left\{u_{n}\left(t_{n j}\right)\right.$ : $1 \leqslant j \leqslant m\}$ have been found we have

$$
u_{n}\left(t_{n}+v h_{n}\right)=\sum_{j=1}^{m} L_{j}(v) u_{n}\left(t_{n j}\right), \quad t_{n}+v h_{n} \in \sigma_{n}(0 \leqslant n \leqslant N-1),
$$

where $L_{j}$ denotes the $j$ th Lagrange fundamental polynomial for the $m$ collocation parameters $\left\{c_{j}\right\}$; i.e.

$$
L_{j}(v):=\prod_{\substack{k=1 \\ k \neq j}}^{m}\left(v-c_{k}\right) /\left(c_{j}-c_{k}\right) \quad(1 \leqslant j \leqslant m) .
$$


We note in passing that the particular choice: $c_{1}=0$ and $c_{m}=1$, implies that the approximating element $u$ will be continuous on the entire interval $I$; that is, $u$ is then an element of the smoother polynomial spline space

$$
S_{m-1}^{(0)}\left(Z_{N}\right):=S_{m-1}^{(-1)}\left(Z_{N}\right) \cap C(I) .
$$

In the following, we shall be interested in studying the attainable order of convergence of $u$ on $I$, as $N \rightarrow \infty$. It is well-known that, were the exact solution $y$ of (1.1) (or (1.2)) in $C^{m}(I)$, then we would obtain, for a uniform mesh (where $\left.h_{n}=h=T N^{-1}\right)$,

$$
\|y-u\|_{\infty}=\mathcal{O}\left(N^{-m}\right) .
$$

Unfortunately, smooth $g$ and $k$ (or $K$ ) in (1.1) (or in (1.2)) lead, for $0<\alpha<1$, to an exact solution $y$ which behaves like $y(t)=\mathcal{O}\left(t^{1-\alpha}\right)$ near $t=0$; it has thus unbounded derivatives at $t=0$ (compare [16], [12], [14], [3]). As a consequence, the collocation approximation $u \in S_{m-1}^{(-1)}\left(Z_{N}\right)$ given by (1.8), with the underlying mesh being the uniform one, satisfies only

$$
\|y-u\|_{\infty}=\mathcal{O}\left(N^{-(1-\alpha)}\right)
$$

and this order is best possible for any $m \geqslant 1$. (Compare also Section 3 below.)

In view of results from classical approximation theory (see, e.g., [22, pp. 409-425]) this disappointing result is no surprise. However, it has been known for some time that by using polynomial spline functions of degree $m-1$ on certain nonuniform meshes tailored to the behavior of the function $f(t)=t^{1-\alpha}$ (so-called graded meshes; cf. Section 4 below) one can restore the convergence behavior shown in (1.12) (compare [17], [2], [6], [26], and [21, pp. 268-296]; related results on the use of graded meshes in numerical quadrature for integrals containing weakly singular functions in their integrands may be found, e.g., in [19] and in [13]).

This idea has recently been employed to devise high-order methods for the numerical solution of Fredholm integral equations of the second kind with weakly singular kernels: see [7] and [20] for studies of product integration methods on graded meshes; [9] and [10] for Galerkin methods; [23] and [25] for collocation methods (compare also [24] for a comprehensive survey). A survey of collocation methods for Fredholm and Volterra integral equations of the second kind with weakly singular kernels, as well as additional references, may also be found in [4].

As far as Volterra integral equations of the forms (1.1) and (1.2) are concerned, [5] presents a study of product integration techniques (extending the functional-analytic techniques used in, e.g., [7], [9], [20]). In order to construct high-order methods on uniform meshes it is necessary to abandon polynomial spline spaces in favor of special nonpolynomial spline spaces reflecting the behavior of the exact solution of (1.1) or (1.2) near $t=0$. This approach has been investigated in [18] (for $\alpha=1 / 2$ ) and in [3].

In the present paper we carry out an analysis of the convergence properties of collocation approximations in $S_{m-1}^{(-1)}\left(Z_{N}\right)$ to the solution of the Volterra integral equations (1.1), (1.2), both for quasi-uniform sequences of meshes and for graded meshes. Moreover, we extend this analysis to the fully discretized version of the collocation equation (1.8) in which the integrals have been approximated by appropriate quadrature processes (note that the above-mentioned analyses for Fredholm integral equations are all based on the assumption that the integrals be evaluated exactly). 
2. The Attainable Order of Convergence. In this section we state the results on the attainable order of convergence of the collocation approximation $u \in S_{m-1}^{(-1)}\left(Z_{N}\right)$ with respect to the two types of mesh sequences mentioned above, assuming that the integrals occurring in (1.8) and (1.9) are known exactly. The fully discretized collocation equation will be investigated in Section 5 . We shall formulate these results for the linear integral equation (1.2) so as not to be burdened with too many technical assumptions; when giving the proofs (in Sections 3 and 4) we shall indicate how these results can be extended to the nonlinear case (1.1).

A sequence of meshes for the interval $I$ is called quasi-uniform if there exists a finite constant $\gamma$ such that, for all $N \in \mathbf{N}$,

$$
h / h^{\prime} \leqslant \gamma
$$

holds (recall the notation introduced in (1.4)). It is easily seen that such a mesh sequence has the property

$$
h_{n} \leqslant h \leqslant \gamma \cdot T N^{-1}, \quad 0 \leqslant n \leqslant N-1(N \in \mathbf{N})
$$

hence, $h=\mathcal{O}\left(N^{-1}\right)$ for any compact interval $I$. This holds, of course, trivially for uniform meshes, where we have $\gamma=1$ and $h_{n}=T N^{-1}$ for all $n$.

THEOREM 2.1. Let the functions $g$ and $K$ in (1.2) satisfy $g \in C^{m}(I)$ and $K \in C^{m}(S)$, with $m \geqslant 1$, and assume that neither function vanishes identically. If $u \in S_{m-1}^{(-1)}\left(Z_{N}\right)$ is the collocation approximation defined by (1.8), and if $y$ denotes the exact solution of (1.2), then

$$
\|y-u\|_{\infty}=\mathcal{O}\left(N^{-(1-\alpha)}\right)
$$

for any quasi-uniform mesh sequence. The exponent $1-\alpha$ in (2.3) is best possible for all $m \geqslant 1$ and for all collocation parameters $\left\{c_{j}\right\}$ with $0 \leqslant c_{1}<\cdots<c_{m} \leqslant 1$.

Consider now graded meshes of the form

$$
t_{n}:=\left(\frac{n}{N}\right)^{r} \cdot T, \quad 0 \leqslant n \leqslant N-1(N \geqslant 2),
$$

where the grading exponent $r \in \mathbf{R}$ will always be assumed to satisfy $r \geqslant 1$. (We again suppress the index showing the dependence of $t_{n}$ on $N$.) For any such mesh we have $0<h_{0}=h^{\prime}<h_{1}<\cdots<h_{N-1}=h$, and, in analogy to (2.2),

$$
h_{n} \leqslant h \leqslant r \cdot T N^{-1}, \quad 0 \leqslant n \leqslant N-1(N \in \mathbf{N}) .
$$

Thus the mesh diameters of a sequence of graded meshes of the form (2.4) behave like $h=\mathcal{O}\left(N^{-1}\right)$ on compact intervals.

THEOREM 2.2. Let the functions $g$ and $K$ in (1.2) satisfy the conditions stated in Theorem 2.1. If $u \in S_{m-1}^{(-1)}\left(Z_{N}\right)$ is the collocation approximation defined by (1.8), and if $y$ denotes the exact solution of (1.2), then

$$
\|y-u\|_{\infty}=\mathcal{O}\left(N^{-m}\right) \text {, }
$$

provided we employ the sequence of graded meshes (2.4) corresponding the the grading exponent

$$
r=m /(1-\alpha) \text {. }
$$

This holds for all collocation parameters $\left\{c_{j}\right\}$ with $0 \leqslant c_{1}<\cdots<c_{m} \leqslant 1$. 
Note that the choice (2.7) for the grading exponent leads to optimal (global) convergence, in the sense that the exponent $m$ in (2.6) cannot be replaced by $m+1$. This agrees, of course, with the well-known result in approximation theory which states that $\mathcal{O}\left(N^{-m}\right)$-convergence is best possible when approximating a function $f \in C^{m}(I)$ in $S_{m-1}^{(-1)}\left(Z_{N}\right)$ or in $S_{m-1}^{(0)}\left(Z_{N}\right)$.

3. Proof of Theorem 2.1: Convergence on Quasi-Uniform Meshes. For $g \in C^{m}(I)$ and $K \in C^{m}(S)$ the (unique) solution of (1.2) is in $C[0, T] \cap C^{m}(0, T]$; more precisely, it has the form

$$
y(t)=g(t)+\sum_{k=1}^{\infty} \psi_{k}(t) \cdot t^{k(1-\alpha)}, \quad t \in I,
$$

where $\psi_{k} \in C^{m}(I)(k \geqslant 1)$, and where the series converges absolutely and uniformly on $I$ (compare [3]; see also [16] and [14]). If $\alpha$ is rational, $\alpha=p / q$ (with $p$ and $q$ coprime), then (3.1) may be written as

$$
y(t)=v_{0}(t)+\sum_{s=1}^{q-1} v_{s}(t) \cdot t^{s(1-\alpha)}, \quad t \in I,
$$

with $v_{s} \in C^{m}(I)(0 \leqslant s \leqslant q-1)$. (See also [12] for the case $\alpha=1 / 2$.) For the sake of simplicity of notation (and, not least, in view of practical applications where one usually encounters the values $\alpha=1 / 2, \alpha=1 / 3$, and $\alpha=2 / 3$ ) we shall give the proofs of Theorem 2.1 and Theorem 2.2 for the case of rational $\alpha$; the generalization of the ideas involved in the subsequent arguments to irrational $\alpha$ is straightforward.

On the initial interval $\sigma_{0}=\left[t_{0}, t_{1}\right]$ (where $t_{0}=0$ ) the exact solution (3.2) is not continuously differentiable (unless $y(t) \equiv 0$; this case has been excluded by assuming $g(t) \not \equiv 0$ and $K(t, s) \not \equiv 0)$. However, since $v_{s} \in C^{m}(I)$, we may write

$$
v_{s}\left(t_{0}+v h_{0}\right)=\sum_{l=1}^{m} c_{0 l}^{(s)} v^{l-1}+h_{0}^{m} R_{0 s}(v), \quad v \in[0,1]
$$

where we have set

$$
c_{0 l}^{(s)}:=v_{s}^{(l-1)}\left(t_{0}\right) h_{0}^{l-1} /(l-1) !
$$

and

$$
R_{0 s}(v):=v_{s}^{(m)}\left(\xi_{0 s}\right) \cdot v^{m} / m ! \quad\left(t_{0}<\xi_{0 s}<t_{0}+v h_{0}\right) .
$$

Thus, by (3.2) (setting $\left.\left(t_{0}+v h_{0}\right)^{s(1-\alpha)}=h_{0}^{s(1-\alpha)} \cdot\left[1+\left(v^{s(1-\alpha)}-1\right)\right]\right)$, we obtain

$$
y\left(t_{0}+v h_{0}\right)=\sum_{l=1}^{m} c_{0 l} v^{l-1}+h_{0}^{1-\alpha} \cdot C_{0}(v)+h_{0}^{m} \cdot R_{0}(v), \quad v \in[0,1],
$$

with

$$
\begin{aligned}
c_{0 l} & :=\sum_{s=0}^{q-1} h_{0}^{s(1-\alpha)} \cdot c_{0 l}^{(s)}, \\
C_{0}(v) & :=\sum_{s=1}^{q-1} h_{0}^{(s-1)(1-\alpha)} \cdot\left(v^{s(1-\alpha)}-1\right) \cdot \sum_{l=1}^{m} c_{0 l}^{(s)} v^{l-1},
\end{aligned}
$$

and

$$
R_{0}(v):=\sum_{s=0}^{q-1} h_{0}^{s(1-\alpha)} \cdot R_{0 s}(v) \cdot v^{s(1-\alpha)}
$$


For $1 \leqslant n \leqslant N-1$ we have, since $y \in C^{m}\left[t_{1}, T\right]\left(t_{1}>0\right)$,

$$
y\left(t_{n}+v h_{n}\right)=\sum_{l=1}^{m} c_{n l} v^{l-1}+h_{n}^{m} \cdot R_{n}(v), \quad t_{n}+v h_{n} \in \sigma_{n},
$$

with

$$
c_{n l}:=y^{(l-1)}\left(t_{n}\right) \cdot h_{n}^{l-1} /(l-1) !
$$

and

$$
R_{n}(v):=y^{(m)}\left(t_{n}+\theta_{n} v h_{n}\right) \cdot v^{m} / m ! \quad\left(0<\theta_{n}<1\right) .
$$

Suppose now that the restriction of the approximation $u \in S_{m-1}^{(-1)}\left(Z_{N}\right)$ to the subinterval $\sigma_{n}$ is given by

$$
u_{n}\left(t_{n}+v h_{n}\right)=\sum_{l=1}^{m} \alpha_{n l} v^{l-1}
$$

Thus, the error $e:=y-u\left(\right.$ with $e_{n}:=y-u_{n}$ denoting its restriction to $\left.\sigma_{n}\right)$ assumes the form

$$
e_{n}\left(t_{n}+v h_{n}\right)=\left\{\begin{array}{l}
\sum_{l=1}^{m} \beta_{0 l} v^{l-1}+h_{0}^{1-\alpha} C_{0}(v)+h_{0}^{m} R_{0}(v) \quad \text { if } n=0 ; \\
\sum_{l=1}^{m} \beta_{n l} v^{l-1}+h_{n}^{m} R_{n}(v) \text { if } 1 \leqslant n \leqslant N-1,
\end{array}\right.
$$

where we have defined $\beta_{n l}:=c_{n l}-\alpha_{n l}(1 \leqslant l \leqslant m ; 0 \leqslant n \leqslant N-1)$.

Subtracting the collocation equation (1.8) (with $k(t, s, y)=K(t, s) y$ ) from the integral equation (1.2) (with $t=t_{n j}$ ), we obtain

$$
\begin{aligned}
e_{n}\left(t_{n j}\right)= & h_{n}^{1-\alpha} \cdot \int_{0}^{c_{j}}\left(c_{j}-v\right)^{-\alpha} \cdot K_{n j}\left(t_{n}+v h_{n}\right) e_{n}\left(t_{n}+v h_{n}\right) d v \\
+ & \sum_{i=0}^{n-1} h_{i}^{1-\alpha} \int_{0}^{1}\left(\frac{t_{n j}-t_{i}}{h_{i}}-v\right)^{-\alpha} \cdot K_{n j}\left(t_{i}+v h_{i}\right) e_{i}\left(t_{i}+v h_{i}\right) d v \\
& (1 \leqslant j \leqslant m ; 0 \leqslant n \leqslant N-1) .
\end{aligned}
$$

Here, we have set $K_{n j}(\cdot):=K\left(t_{n j}, \cdot\right)$. The expressions for the errors $e_{i}$ given in (3.10) can now be used in (3.11) to derive a recurrence relation for the components of the vectors $\beta_{n}:=\left(\beta_{n 1}, \ldots, \beta_{n m}\right)^{T} \in \mathbf{R}^{m}(0 \leqslant n \leqslant N-1)$; it reads

$$
\begin{array}{r}
\sum_{l=1}^{m} \beta_{n l}\left\{c_{j}^{l-1}-h_{n}^{1-\alpha} \int_{0}^{c_{j}}\left(c_{j}-v\right)^{-\alpha} \cdot K_{n j}\left(t_{n}+v h_{n}\right) v^{l-1} d v\right\} \\
=\sum_{i=0}^{n-1} h_{i}^{1-\alpha} \cdot \sum_{l=1}^{m} \beta_{i l} \cdot \int_{0}^{1}\left(\frac{t_{n j}-t_{i}}{h_{i}}-v\right)^{-\alpha} K_{n j}\left(t_{i}+v h_{i}\right) v^{l-1} d v+q_{n j} \\
(1 \leqslant j \leqslant m ; 0 \leqslant n \leqslant N-1),
\end{array}
$$


where the remainder terms $q_{n j}$ are defined by

$$
\begin{aligned}
q_{n j}:= & -h_{n}^{m} R_{n}\left(c_{j}\right)+h_{n}^{1-\alpha} \cdot \int_{0}^{c_{j}}\left(c_{j}-v\right)^{-\alpha} \cdot K_{n j}\left(t_{n}+v h_{n}\right)\left(h_{n}^{m} R_{n}(v)\right) d v \\
& +\sum_{i=1}^{n-1} h_{i}^{1-\alpha} \cdot \int_{0}^{1}\left(\frac{t_{n j}-t_{i}}{h_{i}}-v\right)^{-\alpha} \cdot K_{n j}\left(t_{i}+v h_{i}\right)\left(h_{i}^{m} R_{i}(v)\right) d v \\
& +h_{0}^{1-\alpha} \cdot \int_{0}^{1}\left(\frac{t_{n j}-t_{0}}{h_{0}}\right)^{-\alpha} \cdot K_{n j}\left(t_{0}+v h_{0}\right)\left\{h_{0}^{1-\alpha} C_{0}(v)+h_{0}^{m} R_{0}(v)\right\} d v .
\end{aligned}
$$

For the initial interval $\sigma_{0}$ we obtain, in particular,

$$
\begin{aligned}
\sum_{l=1}^{m} \beta_{0 l} & \left\{c_{j}^{l-1}-h_{0}^{1-\alpha} \cdot \int_{0}^{c_{j}}\left(c_{j}-v\right)^{-\alpha} \cdot K_{0 j}\left(t_{0}+v h_{0}\right) v^{l-1} d v\right\} \\
= & h_{0}^{1-\alpha} \cdot\left\{-C_{0}\left(c_{j}\right)-h_{0}^{m+\alpha-1} \cdot R_{0}\left(c_{j}\right)\right. \\
& \left.+h_{0}^{1-\alpha} \int_{0}^{c_{j}}\left(c_{j}-v\right)^{-\alpha} \cdot K_{0 j}\left(t_{0}+v h_{0}\right)\left(C_{0}(v)+h_{0}^{m+\alpha-1} \cdot R_{0}(v)\right) d v\right\}
\end{aligned}
$$

$$
(1 \leqslant j \leqslant m) \text {. }
$$

We shall now show that, for quasi-uniform mesh sequences, all vectors $\beta_{n}$ have $l_{1}$-norms satisfying

$$
\left\|\beta_{n}\right\|_{1}=\mathcal{O}\left(N^{-(1-\alpha)}\right) \quad(0 \leqslant n \leqslant N-1 ; N \rightarrow \infty, \text { with } N h \leqslant \gamma T) .
$$

This result will then be used in (3.10) to establish the assertion (2.3) of Theorem 2.1.

We begin by observing that, since the kernel $K(t, s)$ is bounded on $S$ and since we have $h_{n}=\mathcal{O}\left(N^{-1}\right)$ for $0 \leqslant n \leqslant N-1$ (recall (2.2)), the matrices $V_{m}-h_{n}^{1-\alpha} \cdot C_{n n}$, with

$$
V_{m}:=\left(c_{j}^{l-1}\right), \quad \text { and } \quad C_{n n}:=\left(\int_{0}^{c_{j}}\left(c_{j}-v\right)^{-\alpha} \cdot K_{n j}\left(t_{n}+v h_{n}\right) v^{l-1} d v\right)
$$

$$
(1 \leqslant j, l \leqslant m)
$$

occurring in (3.12) and (3.14) possess uniformly bounded inverses for all sufficiently large $N$ (note that $V_{m}$ is a Vandermonde matrix corresponding to the collocation parameters $\left\{c_{j}\right\}$ satisfying $0 \leqslant c_{1}<\cdots<c_{m} \leqslant 1$ ). Hence (3.12) and (3.14) define a unique sequence of vectors $\beta_{n}$ for all sufficiently large values of $N$, and there exists a finite constant $C_{0}^{\prime}$ such that

$$
\left\|\left(V_{m}-h_{n}^{1-\alpha} \cdot C_{n n}\right)^{-1}\right\|_{1} \leqslant C_{0}^{\prime}, \quad 0 \leqslant n \leqslant N-1 .
$$

In order to show that the sequence $\left\{\left\|\beta_{n}\right\|_{1}\right\}$ is governed by a generalized Gronwall inequality, we require the following result.

Lemma 3.1. Consider a quasi-uniform sequence of meshes for I. Then, for $0 \leqslant i \leqslant$ $n-1(n \leqslant N-1)$, and for all $\left\{c_{j}\right\}$ with $0 \leqslant c_{1}<\cdots<c_{m} \leqslant 1$, we have,

$$
\int_{0}^{1}\left(\frac{t_{n j}-t_{i}}{h_{i}}-v\right)^{-\alpha} v^{l-1} d v<\frac{\gamma^{\alpha}(1+\gamma)^{\alpha}}{1-\alpha} \cdot(n-i)^{-\alpha} \quad(1 \leqslant j, l \leqslant m) .
$$

Proof of Lemma 3.1. For $i=n-1$, we have

$$
\int_{0}^{1}\left(1+c_{j} \frac{h_{n}}{h_{n-1}}-v\right)^{-\alpha} v^{l-1} d v \leqslant \int_{0}^{1}(1-v)^{-\alpha} d v=1 /(1-\alpha)<\frac{\gamma^{\alpha}(1+\gamma)^{\alpha}}{1-\alpha}
$$

since $\gamma \geqslant 1$. 
Suppose, then, that $i \leqslant n-2$. Since $c_{j} \in[0,1]$, we obtain

$$
\begin{aligned}
I_{n i}(\alpha) & :=\int_{0}^{1}\left(\frac{t_{n j}-t_{i}}{h_{i}}-v\right)^{-\alpha} v^{l-1} d v \leqslant \int_{0}^{1}\left(\frac{t_{n}-t_{i}}{h_{i}}-v\right)^{-\alpha} d v \\
& =\frac{1}{1-\alpha}\left[\left(\frac{t_{n}-t_{i}}{h_{i}}\right)^{1-\alpha}-\left(\frac{t_{n}-t_{i}}{h_{i}}-1\right)^{1-\alpha}\right] \\
& =\frac{1}{1-\alpha}\left(\frac{t_{n}-t_{i}}{h_{i}}\right)^{1-\alpha} \cdot\left[1-\left(1-\left(\frac{t_{n}-t_{i}}{h_{i}}\right)^{-1}\right)\right]^{1-\alpha} .
\end{aligned}
$$

Application of the Mean-Value Theorem yields

$$
\left(1-\left(\frac{t_{n}-t_{i}}{h_{i}}\right)^{-1}\right)^{1-\alpha}=1-(1-\alpha) \cdot\left(\frac{t_{n}-t_{i}}{h_{i}}\right)^{-1} \cdot\left(1-\theta_{n i}\left(\frac{t_{n}-t_{i}}{h_{i}}\right)^{-1}\right)^{-\alpha}
$$

(with $0<\theta_{n i}<1$ ), and we thus find

$$
I_{n i}(\alpha) \leqslant\left(\frac{t_{n}-t_{i}}{h_{i}}\right)^{-\alpha} \cdot\left(1-\theta_{n i} \cdot\left(\frac{t_{n}-t_{i}}{h_{i}}\right)^{-1}\right)^{-\alpha} .
$$

So far, we have not specified the type of mesh sequence containing the points $\left\{t_{n}\right\}$.

Suppose now that the mesh sequence is quasi-uniform. Hence, by (2.1),

$$
\frac{t_{n}-t_{i}}{h_{i}} \geqslant \frac{(n-i) h^{\prime}}{h} \geqslant \gamma^{-1} \cdot(n-i) \text {. }
$$

Moreover, since $i \leqslant n-2$,

$$
\begin{aligned}
1-\theta_{n i} \cdot\left(\frac{t_{n}-t_{i}}{h_{i}}\right)^{-1} & =1-\theta_{n i} \cdot\left(\frac{h_{i}+\cdots+h_{n-1}}{h_{i}}\right)^{-1} \\
& \geqslant 1-\left(\frac{h_{i}+h_{i+1}}{h_{i}}\right)^{-1}=1-\left(1+h_{i+1} / h_{i}\right)^{-1} \\
& \geqslant 1-\left(1+h^{\prime} / h\right)^{-1} \geqslant 1-(1+1 / \gamma)^{-1}=(1+\gamma)^{-1} .
\end{aligned}
$$

Using these results in (3.19) we obtain, for $0<\alpha<1$,

$$
I_{n i}(\alpha) \leqslant \gamma^{\alpha}(1+\gamma)^{\alpha} \cdot(n-i)^{-\alpha}<\frac{\gamma^{\alpha}(1+\gamma)^{\alpha}}{1-\alpha} \cdot(n-i)^{-\dot{\alpha}} .
$$

LEMMA 3.2. Let the assumptions of Lemma 3.1 hold. Then:

$$
\sum_{i=0}^{n-1} h_{i}^{1-\alpha} \cdot \int_{0}^{1}\left(\frac{t_{n j}-t_{i}}{h_{i}}-v\right)^{-\alpha} d v \leqslant T^{1-\alpha} /(1-\alpha), \quad 1 \leqslant n \leqslant N .
$$

Proof of Lemma 3.2. Using the initial argument of the previous proof, we find

$$
\begin{aligned}
& \sum_{i=0}^{n-1} h_{i}^{1-\alpha} \cdot \int_{0}^{1}\left(\frac{t_{n j}-t_{i}}{h_{i}}-v\right)^{-\alpha} d v \leqslant \sum_{i=0}^{n-1} h_{i}^{1-\alpha} \cdot \int_{0}^{1}\left(\frac{t_{n}-t_{i}}{h_{i}}-v\right)^{-\alpha} d v \\
& \quad=\frac{1}{1-\alpha} \cdot \sum_{i=0}^{n-1} h_{i}^{1-\alpha}\left\{\left(\frac{t_{n}-t_{i}}{h_{i}}\right)^{1-\alpha}-\left(\frac{t_{n}-t_{i+1}}{h_{i}}\right)^{1-\alpha}\right\} \\
& =\frac{1}{1-\alpha} \cdot \sum_{i=0}^{n-1}\left\{\left(t_{n}-t_{i}\right)^{1-\alpha}-\left(t_{n}-t_{i+1}\right)^{1-\alpha}\right\}=t_{n}^{1-\alpha} /(1-\alpha) \\
& \leqslant T^{1-\alpha} /(1-\alpha) \text { for } n \leqslant N .
\end{aligned}
$$


Note that (3.20) will also be valid for graded meshes; this fact will be used in Section 4.

We now return to (3.12): for $0 \leqslant i \leqslant n-1$, define the matrices $C_{n i}$ by

$$
C_{n i}:=\left(\int_{0}^{1}\left(\frac{t_{n j}-t_{i}}{h_{i}}-v\right)^{-\alpha} \cdot K_{n j}\left(t_{i}+v h_{i}\right) v^{l-1} d v\right) \quad(1 \leqslant j, l \leqslant m),
$$

and introduce the vectors $q_{n}:=\left(q_{n 1}, \ldots, q_{n m}\right)^{T}$, with components defined in (3.13). Thus, (3.12) can be rewritten as

$$
\beta_{n}=\left(V_{m}-h_{n}^{1-\alpha} C_{n n}\right)^{-1} \cdot\left\{\sum_{i=0}^{n-1} h_{i}^{1-\alpha} \cdot C_{n i} \beta_{i}+q_{n}\right\},
$$

provided $N$ is sufficiently large. If $K_{0}:=\max \{|K(t, s)|:(t, s) \in S\}$ then, by Lemma 3.1 , we find

$$
\left\|C_{n i}\right\|_{1} \leqslant C(\alpha) \cdot(n-i)^{-\alpha}, \quad 0 \leqslant i \leqslant n-1,
$$

where $C(\alpha):=m K_{0} \cdot \gamma^{\alpha}(1+\gamma)^{\alpha} /(1-\alpha)$. This can be used, together with (3.17), to obtain

$$
\left\|\beta_{n}\right\|_{1} \leqslant C_{0} h^{1-\alpha} \cdot \sum_{i=0}^{n-1}(n-i)^{-\alpha} \cdot\left\|\beta_{i}\right\|_{1}+C_{0}^{\prime} \cdot\left\|q_{n}\right\|_{1} \quad(0 \leqslant n \leqslant N-1),
$$

with $C_{0}:=C_{0}^{\prime} \cdot C(\alpha)$. This represents a generalized discrete Gronwall inequality (compare [15], [8], [1]), and it follows that

$$
\left\|\beta_{n}\right\|_{1}=\mathcal{O}\left(\left\|q_{n}\right\|_{1}\right), \quad 0 \leqslant n \leqslant N-1,
$$

since $C_{0}^{\prime}$ (given in (3.17)) is a finite constant, and since $N h \leqslant \gamma T$.

It is clear from (3.13) that the order of $\left\|q_{n}\right\|_{1}$ will essentially be governed by that of the terms $h_{i}^{m} R_{i}(\cdot)$, with $R_{i}(v)$ defined in (3.9) and (3.6); for $i \geqslant 1$ these terms involve the $m$ th derivative of the solution $y$ (if $i=0$ then, by (3.6) and (3.3b), $\left.h_{0}^{m} R_{0}(v)=\mathcal{O}\left(N^{-m}\right)\right)$. It follows from (3.2) and from the Leibniz product rule that this derivative has the form

$$
y^{(m)}(t)=v_{0}^{(m)}(t)+\sum_{s=1}^{q-1} \sum_{k=0}^{m}\left(\begin{array}{c}
m \\
k
\end{array}\right)\left(\begin{array}{c}
s(1-\alpha) \\
k
\end{array}\right) k ! \cdot v_{s}^{(m-k)}(t) \cdot t^{s(1-\alpha)-k},
$$

$$
t>0
$$

Thus, upon setting

$$
\gamma_{m k}^{(s)}:=\left(\begin{array}{c}
m \\
k
\end{array}\right)\left(\begin{array}{c}
s(1-\alpha) \\
k
\end{array}\right) \cdot k !, \quad M_{s \nu}:=\max \left\{\left|v_{s}^{(\nu)}(t)\right|: t \in I\right\},
$$

we are led to

$$
h_{i}^{m} \cdot\left|R_{i}(v)\right| \leqslant h_{i}^{m} \cdot\left\{M_{0 m}+\sum_{s=1}^{q-1} \sum_{k=0}^{m}\left|\gamma_{m k}^{(s)}\right| \cdot M_{s, m-k}\left(t_{i}+\theta_{i} v h_{i}\right)^{s(1-\alpha)-k}\right\} .
$$

This, in turn, reveals that the order of $h_{i}^{m} R_{i}(v)$ will depend on the orders of the products $h_{i}^{m} \cdot t_{i}^{s(1-\alpha)-k}$. To be precise, we state

LEMMA 3.3. Consider any quasi-uniform sequence of meshes for $I$, and assume that $1 \leqslant k \leqslant m$. Then, for $s \geqslant 1$,

$$
h_{i}^{m} \cdot t_{i}^{s(1-\alpha)-k}= \begin{cases}\mathcal{O}\left(N^{-m}\right), & \text { if } s(1-\alpha)-k \geqslant 0 \\ \mathcal{O}\left(N^{-(1-\alpha)}\right), & \text { if } s(1-\alpha)-k<0\end{cases}
$$


Proof of Lemma 3.3. Assume first that $s(1-\alpha)-k \geqslant 0$. The first part of (3.27) then follows trivially since $t_{i}^{s(1-\alpha)-k} \leqslant T^{s(1-\alpha)-k}$ and, by $(2.2), h_{i}=\mathcal{O}\left(N^{-1}\right)$ for $1 \leqslant i \leqslant N-1$.

Now let $s(1-\alpha)-k<0$. In this case we have, for $i \geqslant 1$,

$$
t_{i} \geqslant t_{1}=h_{0} \geqslant h^{\prime} \geqslant h / \gamma \geqslant \gamma^{-1} T N^{-1},
$$

and hence, by (2.2),

$$
\begin{aligned}
h_{i}^{m} \cdot t_{i}^{s(1-\alpha)-k} & \leqslant(\gamma T)^{m} N^{-m} \cdot\left(\gamma^{-1} T\right)^{s(1-\alpha)-k} \cdot N^{-(s(1-\alpha)-k)} \\
& =(\gamma T)^{m} \cdot\left(\gamma^{-1} T\right)^{s(1-\alpha)-k} \cdot N^{-(m+s(1-\alpha)-k)} \\
& \leqslant(\gamma T)^{m} \cdot\left(\gamma^{-1} T\right)^{s(1-\alpha)-k} \cdot N^{-(1-\alpha)}, \quad 1 \leqslant i \leqslant N-1,
\end{aligned}
$$

since $m+s(1-\alpha)-k \geqslant s(1-\alpha) \geqslant 1-\alpha>0$ for all $k$ with $1 \leqslant k \leqslant m, s \geqslant 1$, and $\alpha \in(0,1)$.

Consider again (3.26): for $\alpha \in(0,1)$ there is at least one pair $(s, k)$, with $1 \leqslant k \leqslant m, s \geqslant 1$, for which $s(1-\alpha)-k<0$ (take $(s, k)=(1,1))$. Consequently, (3.26) yields

$$
h_{i}^{m}\left|R_{i}(v)\right|=\mathcal{O}\left(N^{-(1-\alpha)}\right), \quad v \in[0,1](1 \leqslant i \leqslant N-1),
$$

where the exponent cannot be replaced by some $\beta>1-\alpha$.

If we now use the results (3.26), (3.27), (3.20) in (3.13) we verify readily that

$$
\left|q_{n j}\right|=\mathcal{O}\left(N^{-(1-\alpha)}\right), \quad 1 \leqslant j \leqslant m(0 \leqslant n \leqslant N-1),
$$

and hence

$$
\left\|q_{n}\right\|_{1}=\mathcal{O}\left(N^{-(1-\alpha)}\right), \quad 0 \leqslant n \leqslant N-1 .
$$

To bring the proof of Theorem 2.1 to its conclusion we return to (3.10): since (3.24) and (3.29) imply $\left\|\beta_{n}\right\|_{1}=\mathcal{O}\left(N^{-(1-\alpha)}\right)$ for all $n$ we find

$$
\left|e_{n}\left(t_{n}+v h_{n}\right)\right| \leqslant\left\|\beta_{n}\right\|_{1}+\mathcal{O}\left(N^{-(1-\alpha)}\right)=\mathcal{O}\left(N^{-(1-\alpha)}\right) \text {, }
$$

$t_{n}+v h_{n} \in \sigma_{n}, \quad 0 \leqslant n \leqslant N-1$ (as $N \rightarrow \infty, N h \leqslant \gamma T$ ). This is equivalent to (2.3).

We conclude this section with two remarks.

(i) As has been mentioned above, the proof is easily extended to the case of irrational $\alpha$ : this follows from the fact that the infinite series in (3.1) converges absolutely and uniformly on $I$, and by Lemma 3.3 which holds for all $s \geqslant 1$.

(ii) If the given integral equation is nonlinear, i.e. (1.1), then we can use a result due to Lubich [14] which states that if $g(t)$ is of the form $g(t)=G\left(t, t^{1-\alpha}\right)$ near $t=0$, and if $G$ and the kernel $k$ are real analytic functions in a neighborhood of the origin (excluding the trivial cases $g \equiv 0, k \equiv 0$ ), then the exact solution of (1.1) near $t=0$ is given by

$$
y(t)=Y\left(t, t^{1-\alpha}\right),
$$

where $Y$ is a real analytic function in a neighborhood of $(0,0)$. It is then easily seen that by expressing $Y$ as a power series, the solution $y$ near $t=0$ can be written in a form analogous to (3.1). In the corresponding error analysis the role of $K_{n j}\left(t_{i}+v h_{i}\right)$ will then be taken by the partial derivative $\partial k\left(t_{n j}, t_{i}+v h_{i}, y\right) / \partial y$, evaluated at 
some suitable value of $y$ (stemming from the application of the Mean-Value Theorem in the linearization of the error equation); in order that the analogue of (3.17) hold, $\partial k / \partial y$ has to be bounded.

4. Proof of Theorem 2.2: Convergence on Graded Meshes. The proof of Theorem 2.2 proceeds in complete analogy to the one of Theorem 2.1 given in the previous section, except that now we shall obtain a different estimate for $\left\|q_{n}\right\|_{1}$, and hence for $\left\|\beta_{n}\right\|_{1}$ (cf. (3.29) and (3.24)).

Let us begin by stating two simple properties of graded meshes of the form (2.4) with $r \geqslant 1$; namely,

$$
t_{n}=n^{r} \cdot t_{1}, \quad 1 \leqslant n \leqslant N
$$

with

$$
t_{1}=h_{0}=T N^{-r}
$$

and

$$
h / h^{\prime}=N^{r} \cdot\left(1-\left(1-N^{-1}\right)^{r}\right) .
$$

(This last result shows, incidentally, that a sequence of graded meshes with $r>1$ is not quasi-uniform, since $h / h^{\prime} \rightarrow \infty$ as $N \rightarrow \infty$.)

For graded meshes we obtain the following analogue of Lemma 3.1:

LEMMA 4.1. Consider any graded mesh of the form (2.4) and with grading exponent $r \geqslant 1$. Then, for $0 \leqslant i \leqslant n-1 \leqslant N-1$ and for all collocation parameters $\left\{c_{j}\right\}$ satisfying $0 \leqslant c_{1}<\cdots<c_{m} \leqslant 1$,

$$
\int_{0}^{1}\left(\frac{t_{n j}-t_{i}}{h_{i}}-v\right)^{-\alpha} \cdot v^{l-1} d v \leqslant \frac{2^{\alpha}}{1-\alpha} \cdot(n-i)^{-\alpha} \quad(1 \leqslant j, l \leqslant m) .
$$

Proof of Lemma 4.1. The first half of the proof of Lemma 3.1 carries over without any change: there, we have shown that, for $i \leqslant n-2$,

$$
I_{n i}(\alpha):=\int_{0}^{1}\left(\frac{t_{n j}-t_{i}}{h_{i}}-v\right)^{-\alpha} \cdot v^{l-1} d v \leqslant\left(\frac{t_{n}-t_{i}}{h_{i}}\right)^{-\alpha} \cdot\left(1-\theta_{n i} \cdot\left(\frac{t_{n}-t_{i}}{h_{i}}\right)^{-1}\right)^{-\alpha},
$$

with $0<\theta_{n i}<1$. For a graded mesh (2.4) with $r \geqslant 1$ we obtain

$$
\frac{t_{n}-t_{i}}{h_{i}}=\frac{h_{n-1}+\cdots+h_{i}}{h_{i}} \geqslant \frac{(n-i) \cdot h_{i}}{h_{i}}=n-i
$$

since $0<h_{0}<\cdots<h_{N-1}(=h)$. Moreover, since $i \leqslant n-2$, we have

$$
\begin{aligned}
1-\theta_{n i} \cdot\left(\frac{t_{n}-t_{i}}{h_{i}}\right)^{-1} & \geqslant 1-\left(\frac{t_{n}-t_{i}}{h_{i}}\right)^{-1} \geqslant 1-\left(\frac{h_{i}+h_{i+1}}{h_{i}}\right)^{-1} \\
& =1-\left(1+h_{i+1} / h_{i}\right)^{-1} \geqslant 1-\frac{1}{1+1}=1 / 2,
\end{aligned}
$$

and this yields

$$
\left(1-\theta_{n i} \cdot\left(\frac{t_{n}-t_{i}}{h_{i}}\right)^{-1}\right)^{-\alpha} \leqslant 2^{\alpha}<2^{\alpha} /(1-\alpha)
$$

Hence,

$$
I_{n i}(\alpha) \leqslant \frac{2^{\alpha}}{1-\alpha} \cdot(n-i)^{-\alpha}, \quad 0 \leqslant i \leqslant n-1
$$


Since the mesh diameter $h$ of a graded mesh satisfies $h=\mathcal{O}\left(N^{-1}\right)$ (recall (2.5)) we may use again the contraction mapping argument of Section 3 (cf. (3.16) and (3.17)) to show that, for all sufficiently large $N,(3.12)$ defines a unique sequence of vectors $\left\{\beta_{n}:=\left(\beta_{n 1}, \ldots, \beta_{n m}\right)^{T}: 0 \leqslant n \leqslant N-1\right\}$, for which the generalized discrete Gronwall inequality (3.23) holds. Note that here we have made use of Lemma 3.2 which is valid both for quasi-uniform and for graded mesh sequences.

As an immediate consequence, the $l_{1}$-norms of these vectors $\beta_{n}$ satisfy again (3.24). However, the estimate for $h_{i}^{m} R_{i}(v)$ (which will eventually determine the order of $\left.\left\|\beta_{n}\right\|_{1}\right)$ turns out to be rather different than that for quasi-uniform mesh sequences given in (3.28). This is due to the following results.

LEMMA 4.2. Consider a graded mesh of the form (2.4), and assume that the grading exponent $r$ is given by

$$
r=m /(1-\alpha) \text {. }
$$

Then, for $1 \leqslant k \leqslant \nu \leqslant m$ and for $s \geqslant 1$,

$$
h_{i}^{\nu} \cdot t_{i}^{s(1-\alpha)-k} \leqslant c \cdot N^{-\nu}, \quad 1 \leqslant i \leqslant N-1,
$$

where $c:=r^{\nu} \cdot 2^{\nu(r-1)} \cdot T^{\nu-k+s(1-\alpha)}$.

Proof of Lemma 4.2. Since $r \geqslant 1$ we find, using (4.1) and the Mean-Value Theorem,

$$
\begin{aligned}
h_{i} & =t_{i+1}-t_{i}=\left((i+1)^{r}-i^{r}\right) \cdot t_{1}=i^{r} \cdot\left(\left(1+i^{-1}\right)^{r}-1\right) \cdot T N^{-r} \\
& =r \cdot i^{r-1} \cdot\left(1+\theta_{i} \cdot i^{-1}\right)^{r-1} \cdot T N^{-r}, \quad \text { with } 0<\theta_{i}<1,
\end{aligned}
$$

and hence $h_{i} \leqslant r \cdot 2^{r-1} \cdot T \cdot i^{r-1} \cdot N^{-r}$. This yields, again employing (4.1),

$$
\begin{aligned}
h_{i}^{\nu} \cdot t_{i}^{s(1-\alpha)-k} & \leqslant\left(r \cdot 2^{r-1} \cdot T\right)^{\nu} \cdot i^{\nu(r-1)} \cdot N^{-\nu r} \cdot\left(i^{r} \cdot T N^{-r}\right)^{s(1-\alpha)-k} \\
& =c \cdot i^{\nu(r-1)+r s(1-\alpha)-r k} \cdot N^{-\nu r-r s(1-\alpha)+r k} \\
& =c \cdot i^{r(\nu-k)+r s(1-\alpha)-\nu} \cdot N^{-r(\nu-k)-r s(1-\alpha)},
\end{aligned}
$$

with the constant $c$ as defined in Lemma 4.2.

For $r=m /(1-\alpha)$ this reduces to

$$
h_{i}^{\nu} \cdot t_{i}^{s(1-\alpha)-k} \leqslant c \cdot\left(\frac{i}{N}\right)^{r(\nu-k)} \cdot i^{m s-\nu} \cdot N^{-m s},
$$

where the exponent of $i$ satisfies $m s-\nu \geqslant m s-m=m(s-1) \geqslant 0$, since $s \geqslant 1$. It thus follows that, for all $i \leqslant N$, and with $1 \leqslant k \leqslant \nu \leqslant m$,

$$
h_{i}^{\nu} \cdot t_{i}^{s(1-\alpha)-k} \leqslant c \cdot N^{m s-\nu} \cdot N^{-m s}=c \cdot N^{-\nu} .
$$

If we now use the result of Lemma 4.2, with $\nu=m$, in (3.26), we find with no further difficulties the estimate

$$
h_{i}^{m} \cdot\left|R_{i}(v)\right|=\mathcal{O}\left(N^{-m}\right), \quad v \in[0,1](1 \leqslant i \leqslant N-1),
$$

where the exponent $m$ is best possible. By (3.13) this then leads to

$$
\left\|q_{n}\right\|_{1}=\mathcal{O}\left(N^{-m}\right)
$$

since, by (4.1b), $h_{0}=T N^{-r}$; hence $h_{0}^{1-\alpha}=T^{1-\alpha} \cdot N^{-r(1-\alpha)}=T^{1-\alpha} \cdot N^{-m}$. By (3.23) and (3.24) we have thus shown that

$$
\left\|\beta_{n}\right\|_{1}=\mathcal{O}\left(N^{-m}\right), \quad 0 \leqslant n \leqslant N-1(N \rightarrow \infty, N h \leqslant r T),
$$


provided the grading exponent $r$ is as in (4.4). Using once more the expression (3.10) for the error function $e_{n}\left(t_{n}+v h_{n}\right)$, together with the fact that $h_{0}^{1-\alpha}=\mathcal{O}\left(N^{-m}\right)$ for the above grading exponent, we obtain assertion (2.6) of Theorem 2.2.

The above proof is easily modified to deal with the case where, instead of (4.4), we have

$$
r=\mu /(1-\alpha), \quad 1 \leqslant \mu<m .
$$

We now find, for $1 \leqslant k \leqslant \nu \leqslant m$,

$$
\begin{aligned}
h_{i}^{\nu} \cdot t_{i}^{s(1-\alpha)-k} & \leqslant c \cdot\left(\frac{i}{N}\right)^{r(\nu-k)} \cdot i^{r s(1-\alpha)-\nu} \cdot N^{-r s(1-\alpha)} \\
& \leqslant c \cdot i^{\mu s-\nu} N^{-\mu s}, \quad 1 \leqslant i \leqslant N .
\end{aligned}
$$

If $\mu s-\nu \geqslant 0$, then $h_{i}^{\nu} \cdot t_{i}^{s(1-\alpha)-k}=\mathcal{O}\left(N^{-\nu}\right)$. However, since $\mu<m$, we shall also have $\mu s-\nu<0$ for some values of $(s, \nu)$ (e.g., for $(s, \nu)=(1, m))$, in which case the above estimate will no longer be valid. Instead, writing $\mu=m-(m-\mu)$, and observing that $m s-\nu \geqslant 0$ and $m-\mu>0$, we obtain

$$
\begin{aligned}
h_{i}^{\nu} \cdot t_{i}^{s(1-\alpha)-k} & \leqslant c \cdot i^{(m-(m-\mu)) s-\nu} \cdot N^{-(m-(m-\mu)) s} \\
& \leqslant c \cdot N^{m s-\nu} \cdot i^{-(m-\mu) s} \cdot N^{-m s+(m-\mu) s} \\
& \leqslant c \cdot N^{-(\nu-(m-\mu)) s}, \quad 1 \leqslant i \leqslant N(s \geqslant 1) .
\end{aligned}
$$

For the value of $\nu$ relevant in our analysis, $\nu=m$, this becomes

$$
h_{i}^{m} \cdot t_{i}^{s(1-\alpha)-k} \leqslant c \cdot N^{-\mu s}, \quad s \geqslant 1 .
$$

Hence, if the grading exponent $r$ in (2.4) is given by (4.9), then there results the estimate $\left\|q_{n}\right\|_{1}=\mathcal{O}\left(N^{-\mu}\right)$ (note that now $h_{0}^{1-\alpha}=T^{1-\alpha} \cdot N^{-\mu}$ ) and, by (3.24), $\left\|\beta_{n}\right\|_{1}$ $=\mathcal{O}\left(N^{-\mu}\right)(0 \leqslant n \leqslant N-1)$. By (3.10) we then readily establish the following result.

THEOREM 4.1. Let the functions $g$ and $K$ in (1.2) satisfy the smoothness hypotheses stated in Theorem 2.1, and let $u \in S_{m-1}^{(-1)}\left(Z_{N}\right)$ denote the collocation approximation defined by (1.8), with collocation parameters $\left\{c_{j}\right\}$ satisfying $0 \leqslant c_{1}<\cdots<c_{m} \leqslant 1$. Then, for the sequence of graded meshes (2.4) corresponding to the grading exponent $r=\mu /(1-\alpha)(1 \leqslant \mu \leqslant m)$, the collocation error behaves like

$$
\|y-u\|_{\infty}=\mathcal{O}\left(N^{-\mu}\right) \text {. }
$$

In particular, the choice $\mu=1$ (i.e., $r=1 /(1-\alpha)$ ) will yield collocation approximations which, on $I$, converge linearly to the solution $y$ of (1.2), independent of how one selects $m$.

The proofs of the above results are again easily extended to linear integral equations (1.2) with irrational $\alpha$, and to nonlinear integral equations (1.1). We refer to the remarks made at the end of Section 3.

5. Discretization of the Collocation Equation. Until now it has been assumed that the integrals

$$
\Phi_{n i}^{(j)}\left[u_{i}\right]:=\left\{\begin{array}{r}
\int_{0}^{1}\left(\frac{t_{n j}-t_{i}}{h_{i}}-v\right)^{-\alpha} \cdot k\left(t_{n j}, t_{i}+v h_{i}, u_{i}\left(t_{i}+v h_{i}\right)\right) d v, \\
\int_{0}^{c_{j}}\left(c_{j}-v\right)^{-\alpha} \cdot k\left(t_{n j}, t_{n}+v h_{n}, u_{n}\left(t_{n}+v h_{n}\right)\right) d v, \quad i=n-1, \\
\quad(1 \leqslant j \leqslant m)
\end{array}\right.
$$


occurring in the collocation equation (1.8) are known exactly; i.e., that the collocation approximation $u \in S_{m-1}^{(-1)}\left(Z_{N}\right)$ is obtained from what we shall refer to as the exact collocation equation

$$
u_{n}\left(t_{n j}\right)=g\left(t_{n j}\right)+h_{n}^{1-\alpha} \cdot \Phi_{n n}^{(j)}\left[u_{n}\right]+\sum_{i=0}^{n-1} h_{i}^{1-\alpha} \cdot \Phi_{n i}^{(j)}\left[u_{i}\right]
$$

$$
(1 \leqslant j \leqslant m ; 0 \leqslant n \leqslant N-1) .
$$

In practical applications this will rarely be possible, making a further discretization step necessary which will involve numerical quadrature. Suppose, then, that the integrals in (5.1) are approximated by

$$
\hat{\Phi}_{n i}^{(j)}\left[u_{i}\right]:=\left\{\begin{array}{l}
\sum_{l=1}^{\mu_{1}} w_{j l}^{(n, i)}(\alpha) \cdot k\left(t_{n j}, t_{i}+d_{l} h_{i}, u_{i}\left(t_{i}+d_{l} h_{i}\right)\right), \\
0 \leqslant i \leqslant n-1, \quad i=n
\end{array}\right.
$$

$(1 \leqslant j \leqslant m)$

for the case where $c_{1}=0$ we set $\hat{\Phi}_{n n}^{(1)}\left[u_{n}\right]:=0\left(=\Phi_{n n}^{(1)}\left[u_{n}\right]\right)$. It will be assumed that the quadrature abscissas in (5.3) are characterized by the parameters

$$
0 \leqslant d_{1}<\cdots<d_{\mu_{1}} \leqslant 1
$$

and

$$
0 \leqslant d_{j 1}<\cdots<d_{j \mu_{0}} \leqslant c_{j} \quad(1 \leqslant j \leqslant m),
$$

with $\mu_{0} \geqslant 1, \mu_{1} \geqslant 1$ (and, usually, $\mu_{0} \leqslant m, \mu_{1} \leqslant m$ ). (Note that due to the choice (5.4b) the quadrature formulas $\hat{\Phi}_{n n}^{(j)}\left[u_{n}\right]$ will only involve kernel values $k(t, s, \cdot)$ lying in the domain of $k$; in general, it may not be possible to extend $k(t, s, \cdot)$ smoothly to points $(t, s)$ with $s>t$.) Moreover, we shall assume that the quadrature weights in (5.3) are given by

$$
w_{j l}^{(n, i)}(\alpha):=\int_{0}^{1}\left(\frac{t_{n j}-t_{i}}{h_{i}}-v\right)^{-\alpha} \cdot \lambda_{l}(v) d v \quad\left(1 \leqslant l \leqslant \mu_{1} ; 1 \leqslant j \leqslant m\right),
$$

and by

$$
w_{j l}(\alpha):=\int_{0}^{c_{j}}\left(c_{j}-v\right)^{-\alpha} \cdot \lambda_{j l}(v) d v \quad\left(1 \leqslant l \leqslant \mu_{0} ; 1 \leqslant j \leqslant m\right),
$$

where

$$
\lambda_{l}(v):=\prod_{\substack{k=1 \\ k \neq l}}^{\mu_{1}}\left(v-d_{k}\right) /\left(d_{l}-d_{k}\right) \text { and } \lambda_{j l}(v):=\prod_{\substack{k=1 \\ k \neq l}}^{\mu_{0}}\left(v-d_{j k}\right) /\left(d_{j l}-d_{j k}\right)
$$

represent, respectively, the Lagrange fundamental polynomials for the points given in (5.4). In other words, we consider the discretization of the exact collocation equation (5.2) by quadrature formulas based on product integration (compare also [19] and the references listed there).

The fully discretized collocation equation is obtained from (5.2) by replacing the exact integrals (5.1) by the corresponding approximations (5.3). In general, one will now generate an approximation $\hat{u} \in S_{m-1}^{(-1)}\left(Z_{N}\right)$ which will be different from the one 
defined by the exact collocation (5.2); i.e., $\hat{u}$ will be given by

$$
\begin{aligned}
\hat{u}_{n}\left(t_{n j}\right)=g\left(t_{n j}\right)+h_{n}^{1-\alpha} \cdot \hat{\Phi}_{n n}^{(j)}\left[\hat{u}_{n}\right]+ & \sum_{i=0}^{n-1} h_{i}^{1-\alpha} \cdot \hat{\Phi}_{n i}^{(j)}\left[\hat{u}_{i}\right] \\
& (1 \leqslant j \leqslant m ; 0 \leqslant n \leqslant N-1),
\end{aligned}
$$

where, in analogy to (1.10), we write

$$
\hat{u}_{n}\left(t_{n}+v h_{n}\right)=\sum_{j=1}^{m} L_{j}(v) \cdot \hat{u}_{n}\left(t_{n j}\right), \quad t_{n}+v h_{n} \in \sigma_{n} .
$$

Setting $\hat{e}:=y-\hat{u}, e:=y-u$, and $\varepsilon:=u-\hat{u}$, it follows from $\hat{e}=(y-u)+$ $(u-\hat{u})$ that

$$
\|\hat{e}\|_{\infty} \leqslant\|e\|_{\infty}+\|\varepsilon\|_{\infty} .
$$

Global convergence results for $\hat{u}$ will thus be obtained by estimating the order of the perturbation $\varepsilon$ due to the full discretization of (5.2), and by using the results on the behavior of $e$ derived in the previous sections. For simplicity, we shall state the results again for the linear equation (1.2); according to the remark at the end of Section 3, their extension to nonlinear equations is straightforward.

THEOREM 5.1. Let $g$ and $K$ in (1.2) be $m$ times continuously differentiable on their respective domains. Assume that $u, \hat{u} \in S_{m-1}^{(-1)}\left(Z_{N}\right)$ denote the solution of the exact collocation equation (5.2) and that of its fully discretized version (5.6), where the quadrature formulas (5.3), satisfying (5.4) and (5.5), have been used. Then the perturbation $\varepsilon:=u-\hat{u}$ behaves like

$$
\|\varepsilon\|_{\infty}=\mathcal{O}\left(N^{-\mu}\right)
$$

where $\mu:=\min \left(\mu_{0}+1-\alpha, \mu_{1}\right)$, and this holds for quasi-uniform sequences of meshes as well as for graded mesh sequences (2.4) with $r \geqslant 1$.

Proof. Let

$$
E_{n i}^{(j)}\left[u_{i}\right]:=\Phi_{n i}^{(j)}\left[u_{i}\right]-\hat{\Phi}_{n i}^{(j)}\left[u_{i}\right] .
$$

Hence, subtracting (5.6) from (5.2) and setting $k(t, s, y)=K(t, s) \cdot y$, we obtain

$$
\begin{aligned}
\varepsilon_{n}\left(t_{n j}\right)= & h_{n}^{1-\alpha} \cdot \hat{\Phi}_{n n}^{(j)}\left[\varepsilon_{n}\right]+\sum_{i=0}^{n-1} h_{i}^{1-\alpha} \cdot \hat{\Phi}_{n i}^{(j)}\left[\varepsilon_{i}\right] \\
& +\sum_{i=0}^{n} h_{i}^{1-\alpha} \cdot E_{n i}^{(j)}\left[u_{i}\right] \quad(1 \leqslant j \leqslant m ; 0 \leqslant n \leqslant N-1),
\end{aligned}
$$

where $\varepsilon_{n}(t)$ denotes the restriction of $\varepsilon(t)$ to the subinterval $\sigma_{n}$. Since $\varepsilon_{n} \in \pi_{m-1}$ we may write

$$
\varepsilon_{n}\left(t_{n}+v h_{n}\right)=\sum_{l=1}^{m} L_{l}(v) \cdot \varepsilon_{n}\left(t_{n}+c_{l} h_{n}\right), \quad t_{n}+v h_{n} \in \sigma_{n}
$$


with $L_{l}(v)$ representing the $l$ th Lagrange fundamental polynomial associated with the $m$ collocation parameters. The terms $\hat{\Phi}_{n i}^{(j)}\left[\varepsilon_{i}\right]$ in (5.11) are thus of the form

$\hat{\boldsymbol{\Phi}}_{n i}^{(j)}\left[\varepsilon_{i}\right]= \begin{cases}\sum_{l=1}^{m}\left(\sum_{s=1}^{\mu_{1}} w_{j s}^{(n, i)}(\alpha) \cdot K_{n j}\left(t_{i}+d_{s} h_{i}\right) \cdot L_{l}\left(d_{s}\right)\right) \cdot \varepsilon_{i}\left(t_{i}+c_{l} h_{i}\right), & i<n, \\ \sum_{l=1}^{m}\left(\sum_{s=1}^{\mu_{0}} w_{j s}(\alpha) \cdot K_{n j}\left(t_{n}+d_{j s} h_{n}\right) \cdot L_{l}\left(d_{j s}\right)\right) \cdot \varepsilon_{n}\left(t_{n}+c_{l} h_{n}\right), & i=n\end{cases}$ $(1 \leqslant j \leqslant m)$.

Let $Q_{n i}(\alpha) \quad(0 \leqslant i \leqslant n \leqslant N-1)$ denote the square matrix of order $m$ whose elements are

$$
q_{j l}^{(n, i)}(\alpha):= \begin{cases}\sum_{s=1}^{\mu_{1}} w_{j s}^{(n, i)}(\alpha) \cdot K_{n j}\left(t_{i}+d_{s} h_{i}\right) \cdot L_{l}\left(d_{s}\right), & 0 \leqslant i \leqslant n-1, \\ \sum_{s=1}^{\mu_{0}} w_{j s}(\alpha) \cdot K_{n j}\left(t_{n}+d_{j s} h_{n}\right) L_{l}\left(d_{j s}\right), & i=n\end{cases}
$$

$(1 \leqslant j, l \leqslant m)$

and define the vectors

$$
\begin{aligned}
r_{n i} & :=\left(E_{n i}^{(1)}\left[u_{i}\right], \ldots, E_{n i}^{(m)}\left[u_{i}\right]\right)^{T}, \\
\eta_{i} & :=\left(\varepsilon_{i}\left(t_{i}+c_{1} h_{i}\right), \ldots, \varepsilon_{i}\left(t_{i}+c_{m} h_{i}\right)\right)^{T} .
\end{aligned}
$$

With this notation, Eq. (5.11) can be expressed in the form

$$
\begin{aligned}
\left(I_{m}-h_{n}^{1-\alpha} \cdot Q_{n n}(\alpha)\right) \cdot \eta_{n}=\sum_{i=0}^{n-1} h_{i}^{1-\alpha} \cdot Q_{n i}(\alpha) \eta_{i}+\sum_{i=0}^{n} h_{i}^{1-\alpha} \cdot r_{n i} & (0 \leqslant n \leqslant N-1),
\end{aligned}
$$

where $I_{m}$ is the identity matrix of order $m$. Consider the matrix multiplying $\eta_{n}$ : since the elements of $Q_{n n}(\alpha)$ are bounded (this follows from the boundedness of the kernel $K(t, s)$ and from that of the quadrature weights $(5.5 \mathrm{~b}))$, and since $h_{n}=\mathcal{O}\left(N^{-1}\right)$ $(n \leqslant N-1)$ both for quasi-uniform and for graded mesh sequences (recall (2.2) and (2.5)), there exists a finite constant $Q_{0}^{\prime}$ such that, for all sufficiently large $N$,

$$
\left\|\left(I_{m}-h_{n}^{1-\alpha} \cdot Q_{n n}(\alpha)\right)^{-1}\right\|_{1} \leqslant Q_{0}^{\prime}, \quad 0 \leqslant n \leqslant N-1 .
$$

In order to show that the $l_{1}$-norms of the vectors $\eta_{n}$ are governed, in analogy to (3.23), by a generalized discrete Gronwall inequality we require the following

LEMMA 5.1. The quadrature weights $w_{j l}^{(n, i)}(\alpha)(i<n)$ defined by (5.5a) satisfy

$$
\left|w_{j l}^{(n, i)}(\alpha)\right| \leqslant w(\alpha) \cdot(n-i)^{-\alpha} \quad\left(1 \leqslant j \leqslant m ; 1 \leqslant l \leqslant \mu_{1}\right),
$$

where the constant $w(\alpha)$ is given by

$$
w(\alpha):=\left\{\begin{array}{l}
\frac{\gamma^{\alpha}(1+\gamma)^{\alpha}}{1-\alpha} \cdot \Lambda_{1} \quad \text { for quasi-uniform meshes } \\
\frac{2^{\alpha}}{1-\alpha} \cdot \Lambda_{1} \quad \text { for graded meshes }(2.4) ;
\end{array}\right.
$$

here, $\Lambda_{1}:=\max \left\{\sum_{l=1}^{\mu_{1}}\left|\lambda_{l}(v)\right|: v \in[0,1]\right\}$ denotes the Lebesgue constant associated with the quadrature parameters $\left\{d_{1}, \ldots, d_{\mu_{1}}\right\}$. 
The proof of this assertion is an immediate consequence of Lemma 3.1 (for quasi-uniform mesh sequences) and of Lemma 4.1 (for graded meshes).

The above lemma allows us to derive bounds for the norms $\left\|Q_{n i}(\alpha)\right\|_{1}$ : using (5.13) we find

$$
\begin{aligned}
\left\|Q_{n i}(\alpha)\right\|_{1} & =\max \left\{\sum_{j=1}^{m}\left|q_{j l}^{(n, i)}(\alpha)\right|: 1 \leqslant l \leqslant m\right\} \\
& \leqslant Q(\alpha) \cdot(n-i)^{-\alpha}, \quad 0 \leqslant i \leqslant n-1(n \leqslant N-1),
\end{aligned}
$$

with the constant $Q(\alpha)$ depending on the bound for $K(t, s)$ and on the Lebesgue constant $\Lambda_{1}$. Applying the above results in (5.14) we obtain

$$
\begin{aligned}
\left\|\eta_{n}\right\|_{1} \leqslant Q_{0} \cdot h^{1-\alpha} \cdot \sum_{i=0}^{n-1}(n-i)^{-\alpha} \cdot\left\|\eta_{i}\right\|_{1}+Q_{0}^{\prime} \cdot \sum_{i=0}^{n} h_{i}^{1-\alpha} \cdot\left\|r_{n i}\right\|_{1} \\
\quad(0 \leqslant n \leqslant N-1),
\end{aligned}
$$

with $Q_{0}:=Q_{0}^{\prime} \cdot Q(\alpha)$. This is the desired generalized discrete Gronwall inequality; in analogy to (3.24), the order of the quantities $\left\|\eta_{n}\right\|_{1}$ will be given by the order of the terms $\sum_{i=0}^{n} h_{i}^{1-\alpha} \cdot\left\|r_{n i}\right\|_{1}$.

LEMMA 5.2. Let the assumptions of Theorem 5.1 hold. Then we have

$$
\sum_{i=0}^{n} h_{i}^{1-\alpha} \cdot\left\|r_{n i}\right\|_{1}=\mathcal{O}\left(N^{-\mu}\right), \quad 0 \leqslant n \leqslant N-1,
$$

with $\mu:=\min \left(\mu_{0}+1-\alpha, \mu_{1}\right)$, independent of whether we consider quasi-uniform or graded mesh sequences.

Proof of Lemma 5.2. Recall that the components of $r_{n i}$ are the quadrature errors introduced in (5.10). According to the hypotheses imposed on the quadrature formulas (5.3) these quadrature errors are bounded; specifically, we have

$$
\left|E_{n i}^{(j)}\left[u_{i}\right]\right| \leqslant\left\{\begin{array}{l}
\gamma_{1} h_{i}^{\mu_{1}} \cdot \int_{0}^{1}\left(\frac{t_{n j}-t_{i}}{h_{i}}-v\right)^{-\alpha} d v \quad \text { if } i \leqslant n-1, \\
\gamma_{0} h_{i}^{\mu_{0}} \cdot \int_{0}^{c_{j}}\left(c_{j}-v\right)^{-\alpha} d v \quad \text { if } i=n \quad(1 \leqslant j \leqslant m),
\end{array}\right.
$$

with $\gamma_{0}$ and $\gamma_{1}$ denoting suitable constants. To show this, let $i<n$,

$$
\phi_{n j}\left(t_{i}+v h_{i}\right):=K_{n j}\left(t_{i}+v h_{i}\right) u_{i}\left(t_{i}+v h_{i}\right)
$$

and denote by $\psi_{n j}\left(t_{i}+v h_{i}\right)$ the interpolating polynomial (of degree $\mu_{1}$ ) for $\phi_{n j}$ with respect to the points $\left\{t_{i}+d_{s} h_{i}: 1 \leqslant s \leqslant \mu_{1}\right\}$. Since $\phi_{n j}$ has continuous derivatives of order $m$ on $\sigma_{i}$, the interpolation error has the form

$$
\phi_{n j}\left(t_{i}+v h_{i}\right)-\psi_{n j}\left(t_{i}+v h_{i}\right)=\phi_{n j}^{\left(\mu_{1}\right)}\left(\xi_{i}\right) \cdot h_{i}^{\mu_{1}} \cdot \prod_{s=1}^{\mu_{1}}\left(v-d_{s}\right) / \mu_{1} !,
$$

with $\xi_{i} \in \sigma_{i}$, and for all $\mu_{1} \leqslant m$. An analogous expression holds when $i=n$, with $\mu_{1}$ replaced by $\mu_{0}$. According to $(5.5), E_{n i}^{(j)}\left[u_{i}\right]$ is equal to the weighted integral of the above interpolation error, with weight functions as in (5.5); from this, (5.19) follows immediately. 
We thus obtain, setting first $i \leqslant n-1$ and using $t_{n j}-t_{i} \geqslant t_{n}-t_{i}$,

$$
\begin{aligned}
\left\|r_{n i}\right\|_{1} & =\sum_{j=1}^{m}\left|E_{n i}^{(j)}\left[u_{i}\right]\right| \leqslant m \cdot \gamma_{1} h_{i}^{\mu_{1}} \cdot \int_{0}^{1}\left(\frac{t_{n}-t_{i}}{h_{i}}-v\right)^{-\alpha} d v \\
& =\frac{m \cdot \gamma_{1} h_{i}^{\mu_{1}}}{1-\alpha} \cdot h_{i}^{\alpha-1} \cdot\left\{\left(t_{n}-t_{i}\right)^{1-\alpha}-\left(t_{n}-t_{i+1}\right)^{1-\alpha}\right\}
\end{aligned}
$$

if $i=n$ we find

$$
\left\|r_{n n}\right\|_{1} \leqslant \frac{m \cdot \gamma_{0} h_{n}^{\mu_{0}}}{1-\alpha}
$$

It now follows that

$$
\begin{aligned}
& \sum_{i=0}^{n} h_{i}^{1-\alpha} \cdot\left\|r_{n i}\right\|_{1}=h_{n}^{1-\alpha} \cdot\left\|r_{n n}\right\|_{1}+\sum_{i=0}^{n-1} h_{i}^{1-\alpha}\left\|r_{n i}\right\|_{1} \\
& \quad \leqslant \gamma(\alpha)\left\{h^{\mu_{0}+1-\alpha}+h^{\mu_{1}} \sum_{i=0}^{n-1}\left\{\left(t_{n}-t_{i}\right)^{1-\alpha}-\left(t_{n}-t_{i+1}\right)^{1-\alpha}\right\}\right\} \\
& \quad \leqslant \gamma(\alpha)\left\{h^{\mu_{0}+1-\alpha}+h^{\mu_{1}} t_{n}^{1-\alpha}\right\} \leqslant \gamma(\alpha) \cdot h^{\mu} \cdot\left\{h^{\mu_{0}+1-\alpha-\mu}+T^{1-\alpha} \cdot h^{\mu_{1}-\mu}\right\},
\end{aligned}
$$

with $\gamma(\alpha):=\max \left(m \cdot \gamma_{0} /(1-\alpha), m \cdot \gamma_{1} /(1-\alpha)\right)$, and with $\mu$ defined as in Lemma 5.2. Since the factor multiplying $h^{\mu}$ is uniformly bounded, we have established (5.18).

We now return to the Gronwall inequality (5.17): since the mesh diameter $h$ satisfies $h=\mathcal{O}\left(N^{-1}\right)$ for both types of mesh sequences considered here, (5.17) implies $\left\|\eta_{n}\right\|_{1}=\mathcal{O}\left(N^{-\mu}\right)$, on the basis of the above result. On the other hand, (5.12) leads to

$$
\left|\varepsilon_{n}\left(t_{n}+v h_{n}\right)\right| \leqslant\left\|L_{l}\right\|_{\infty} \cdot\left\|\eta_{n}\right\|_{1} \leqslant \Lambda \cdot\left\|\eta_{n}\right\|_{1}, \quad t_{n}+v h_{n} \in \sigma_{n}(0 \leqslant n \leqslant N-1),
$$

and the result of Theorem 5.1 follows, since the Lebesgue constant $\Lambda$ corresponding to the (fixed number) $m$ of collocation parameters $\left\{c_{j}\right\}$ is bounded.

We are now in a position to derive our results on the attainable order of convergence of the approximation $\hat{u} \in S_{m-1}^{(-1)}\left(Z_{N}\right)$ defined by the fully discretized collocation equation (5.6); since quasi-uniform mesh sequences are of no interest in practical applications (recall Theorem 2.1), we shall state only the result for graded meshes. The proof of the following theorem is, of course, a direct consequence of (5.8), Theorem 2.2, and Theorem 5.1.

THEOREM 5.2. Let $g$ and $K$ in (1.2) be $m$ times continuously differentiable on their respective domains $I$ and $S$, and let $\hat{u} \in S_{m-1}^{(-1)}\left(Z_{N}\right)$ denote the solution of the fully discretized collocation equation (5.6). Moreover, assume that the quadrature approximations (5.3) used in (5.6) correspond to

$$
\mu_{0}=\mu_{1}=m, \quad d_{l}=c_{l}, \quad d_{j l}=c_{j} c_{l} \quad(1 \leqslant j, l \leqslant m) .
$$

Then for the graded mesh (2.4) with grading exponent $r=m /(1-\alpha)$ we have

$$
\|y-\hat{u}\|_{\infty}=\mathcal{O}\left(N^{-m}\right) \text {. }
$$


It is clear that the above result can be generalized to cover the cases where the quadrature parameters (5.4) are not related to the collocation parameters $\left\{c_{j}\right\}$, and where the grading exponent is given the value $r=\mu /(1-\alpha), 1 \leqslant \mu \leqslant m$ (compare Theorem 4.1). By (5.8), Theorem 5.1, and Theorem 4.1, the corresponding results are obvious, and we therefore refrain from stating them explicitly.

6. An Example. Consider the fully discretized collocation equation (5.6) where the quadratures $\hat{\Phi}_{n i}^{(j)}\left[\hat{u}_{i}\right]$ are characterized by $\mu_{0}=\mu_{1}=m, d_{l}=c_{l}, d_{j l}=c_{j} c_{l}(1 \leqslant j, l \leqslant$ $m)$. Setting

$$
\hat{Y}_{i s}:=\hat{u}_{i}\left(t_{i}+c_{s} h_{i}\right) \quad(1 \leqslant s \leqslant m),
$$

and using (5.7), the quadrature approximations in (5.6) assume the form

$$
\hat{\Phi}_{n i}^{(j)}\left[\hat{u}_{i}\right]=\left\{\begin{array}{l}
\sum_{l=1}^{m} w_{j l}^{(n, i)}(\alpha) \cdot k\left(t_{n j}, t_{i}+c_{l} h_{i}, \hat{Y}_{i l}\right) \quad \text { if } 0 \leqslant i \leqslant n-1, \\
\sum_{l=1}^{m} w_{j l}(\alpha) \cdot k\left(t_{n j}, t_{n}+c_{j} c_{l} h_{n}, \sum_{s=1}^{m} L_{s}\left(c_{j} c_{l}\right) \cdot \hat{Y}_{n s}\right) \quad \text { if } i=n,
\end{array}\right.
$$

with $t_{n j}:=t_{n}+c_{j} h_{n}(1 \leqslant j \leqslant m)$. In the expressions (5.5) for the quadrature weights we now have $\lambda_{l}(v)=L_{l}(v)$, and hence the above weights become

$$
w_{j l}^{(n, i)}(\alpha)=\int_{0}^{1}\left(\frac{t_{n j}-t_{i}}{h_{i}}-v\right)^{-\alpha} \cdot L_{l}(v) d v
$$

and

$$
w_{j l}(\alpha)=\int_{0}^{c_{j}}\left(c_{j}-v\right)^{-\alpha} \cdot \prod_{\substack{k=1 \\ k \neq l}}^{m}\left(v-c_{j} c_{k}\right) /\left(c_{j}\left(c_{l}-c_{k}\right)\right) d v \quad(1 \leqslant j, l \leqslant m) .
$$

This last expression can be simplified by an obvious substitution; we find

$$
w_{j l}(\alpha)=c_{j}^{1-\alpha} \cdot \int_{0}^{1}(1-v)^{-\alpha} \cdot L_{l}(v) d v .
$$

Thus, according to (5.6) and (6.1), the fully discretized collocation equation,

$$
\hat{Y}_{n j}=\hat{F}_{n}\left(\hat{u} ; t_{n j}\right)+h_{n}^{1-\alpha} \cdot \hat{\Phi}_{n n}^{(j)}\left[\hat{u}_{n}\right] \quad(1 \leqslant j \leqslant m)
$$

with

$$
\hat{F}_{n}\left(\hat{u} ; t_{n j}\right):=g\left(t_{n j}\right)+\sum_{i=0}^{n-1} h_{i}^{1-\alpha} \cdot \hat{\Phi}_{n i}^{(j)}\left[\hat{u}_{i}\right] \quad(0 \leqslant n \leqslant N-1),
$$

consititutes, for each $n$, a system of $m$ nonlinear algebraic equations for $\left\{\hat{Y}_{n 1}, \ldots, \hat{Y}_{n m}\right\}$; once these values have been determined, the approximation $\hat{u}$ on $\sigma_{n}$ is given by

$$
\hat{u}_{n}\left(t_{n}+v h_{n}\right)=\sum_{j=1}^{m} L_{j}(v) \cdot \hat{Y}_{n j}
$$


For $m=2$ (i.e., $\hat{u} \in S_{1}^{(-1)}\left(Z_{N}\right)$ ), the quadrature weights (6.2) are:

$$
\begin{aligned}
w_{j 1}(\alpha)= & \frac{c_{j}^{1-\alpha} \cdot\left((2-\alpha) c_{2}-1\right)}{(1-\alpha)(2-\alpha)\left(c_{2}-c_{1}\right)}, \quad w_{j 2}(\alpha)=\frac{c_{j}^{1-\alpha} \cdot\left(1-(2-\alpha) c_{1}\right)}{(1-\alpha)(2-\alpha)\left(c_{2}-c_{1}\right)} ; \\
w_{j 1}^{(n, i)}(\alpha)= & \frac{1}{(1-\alpha)(2-\alpha)\left(c_{2}-c_{1}\right)} \\
& \cdot\left\{\left(\frac{t_{n j}-t_{i}}{h_{i}}\right)^{1-\alpha} \cdot\left((2-\alpha) c_{2}-\frac{t_{n j}-t_{i}}{h_{i}}\right)\right. \\
w_{j 2}^{(n, i)}(\alpha)= & \left.\left.\frac{1}{(1-\alpha)(2-\alpha)\left(c_{2}-c_{1}\right)} h_{n j}-t_{i+1}\right)^{1-\alpha} \cdot\left((2-\alpha)\left(c_{2}-1\right)-\frac{t_{n j}-t_{i+1}}{h_{i}}\right)\right\}, \\
& \cdot\left\{\left(\frac{t_{n j}-t_{i}}{h_{i}}\right)^{1-\alpha} \cdot\left(\frac{t_{n j}-t_{i}}{h_{i}}-(2-\alpha) c_{1}\right)\right. \\
\left.-\left(\frac{t_{n j}-t_{i+1}}{h_{i}}\right)^{1-\alpha} \cdot\left(\frac{t_{n j}-t_{i+1}}{h_{i}}-(2-\alpha)\left(c_{1}-1\right)\right)\right\} & (j=1,2 ; 0 \leqslant i \leqslant n-1) .
\end{aligned}
$$

The corresponding fully discretized collocation equation reads

$$
\begin{aligned}
\hat{Y}_{n j}= & \hat{F}_{n}\left(\hat{u} ; t_{n j}\right) \\
+ & h_{n}^{1-\alpha} \cdot\left\{w_{j 1}(\alpha) \cdot k\left(t_{n j}, t_{n}+c_{j} c_{1} h_{n}, L_{1}\left(c_{j} c_{1}\right) \hat{Y}_{n 1}+L_{2}\left(c_{j} c_{1}\right) \hat{Y}_{n 2}\right)\right. \\
& \left.+w_{j 2}(\alpha) \cdot k\left(t_{n j}, t_{n}+c_{j} c_{2} h_{n}, L_{1}\left(c_{j} c_{2}\right) \hat{Y}_{n 1}+L_{2}\left(c_{j} c_{2}\right) \hat{Y}_{n 2}\right)\right\} \\
& \quad(j=1,2),
\end{aligned}
$$

with

$$
\begin{array}{r}
\hat{F}_{n}\left(\hat{u} ; t_{n j}\right)=g\left(t_{n j}\right)+\sum_{i=0}^{n-1} h_{i}^{1-\alpha} \cdot\left\{w_{j 1}^{(n, i)}(\alpha) \cdot k\left(t_{n j}, t_{i}+c_{1} h_{i}, \hat{Y}_{i 1}\right)\right. \\
\left.+w_{j 2}^{(n, i)}(\alpha) \cdot k\left(t_{n j}, t_{i}+c_{2} h_{i}, \hat{Y}_{i 2}\right)\right\} \\
(0 \leqslant n \leqslant N-1) .
\end{array}
$$

For the graded mesh $t_{n}=(n / N)^{r} \cdot T(0 \leqslant n \leqslant N)$, we obtain

$$
\|y-\hat{u}\|_{\infty}= \begin{cases}\mathcal{O}\left(N^{-2}\right), & \text { if } r=2 /(1-\alpha), \\ \mathcal{O}\left(N^{-1}\right), & \text { if } r=1 /(1-\alpha), \\ \mathcal{O}\left(N^{-(1-\alpha)}\right), & \text { if } r=1 \text { (uniform mesh). }\end{cases}
$$

Acknowledgment. The author gratefully acknowledges the generous hospitality extended to him by CWI (formerly Mathematisch Centrum) at Amsterdam during a recent visit where part of this work was carried out. 
1. P. R. BeESACK, "More generalised discrete Gronwall inequalities," Preprint, 1983.

2. C. DE BOoR, "Good approximation by splines with variable knots," in Spline Functions and Approximation Theory (A. Meir and A. Sharma, eds.), Birkhäuser-Verlag, Basel, 1973, pp. 57-72.

3. H. BRUNNER, "Nonpolynomial spline collocation for Volterra equations with weakly singular kernels,” SIAM J. Numer. Anal., v. 20, 1983, pp. 1106-1119.

4. H. BRUnNer, "The numerical solution of integral equations with weakly singular kernels," in Numerical Analysis, Dundee 1983 (D. F. Griffiths, ed.), Lecture Notes in Math., Vol. 1066, Springer-Verlag, Berlin, 1984, pp. 50-71.

5. H. Brunner \& I. G. Graham, "Product integration for weakly singular Volterra integral equations." (To appear.)

6. H. G. BURCHARD, "On the degree of convergence of piecewise polynomial approximation on optimal meshes. II," Trans. Amer. Math. Soc., v. 234, 1977, pp. 531-559.

7. G. A. Chandler, Superconvergence of Numerical Methods to Second Kind Integral Equations, Ph. D. Thesis, Australian National University, Canberra, 1979.

8. J. Dixon \& S. McKeE, Singular Gronwall Inequalities. Numerical Analysis Report NA/83/44. Hertford College, University of Oxford, 1983.

9. I. G. Graham, The Numerical Solution of Fredholm Integral Equations of the Second Kind, Ph. D. Thesis, University of New South Wales, Kensington, 1980.

10. I. G. Graham, "Galerkin methods for second kind integral equations with singularities", Math. Comp., v. 39, 1982, pp. 519-533.

11. I. G. Graham, Estimates for the Modulus of Smoothness, Research Report No. 22, Dept. of Mathematics, University of Melbourne, 1982.

12. F. DE HoOG \& R. WEISs, "On the solution of a Volterra integral equation with a weakly singular kernel," SIAM J. Math. Anal., v. 4, 1973, pp. 561-573.

13. R. B. Kearfott, “A sinc approximation for the indefinite integral," Math. Comp., v. 41, 1983, pp. $559-572$.

14. Сн. Lubich, "Runge-Kutta theory for Volterra and Abel integral equations of the second kind," Math. Comp., v. 41, 1983, pp. 87-102.

15. S. MCKeE, "Generalised discrete Gronwall lemmas," Z. Angew. Math. Mech., v. 62, 1982, pp. 429-434.

16. R. K. Miller and A. Feldstein, "Smoothness of solutions of Volterra integral equations with weakly singular kernels," SIAM J. Math. Anal., v. 2, 1971, pp. 242-258.

17. J. R. Rice, "On the degree of convergence of nonlinear spline approximation," in Approximation with Special Emphasis on Spline Functions (I. J. Schoenberg, ed.), Academic Press, New York, 1969, pp. 349-365.

18. H. J. J. TE RIELE, “Collocation methods for weakly singular second-kind Volterra integral equations with non-smooth solution," IMA J. Numer. Anal., v. 2, 1982, pp. 437-449.

19. C. SCHNeIder, "Produktintegration mit nicht-äquidistanten Stützstellen," Numer. Math., v. 35 , 1980, pp. $35-43$.

20. C. SCHNeider, "Product integration for weakly singular integral equations," Math. Comp., v. 36, 1981, pp. 207-213.

21. L. L. Schumaker, Spline Functions: Basic Theory, Wiley, New York, 1981.

22. A. F. Timan, Theory of Approximation of Functions of a Real Variable, Pergamon Press, Oxford, 1963.

23. P. R. UBA, "The method of piecewise-linear collocation on a nonuniform grid for the solution of integral equations with a singularity,"Uchen. Zap. Tartu. Gos. Univ'., v. 580, 1981, pp. 52-57. (Russian)

24. G. Vainikxo, A. Pedas \& P. Uba, Methods for Solving Weakly Singular Integral Equations, Tartu. Gos. Univ., Tartu, 1984. (Russian)

25. G. VAINIKKO \& P. UBA, “A piecewise polynomial approximation to the solution of an integral equation with weakly singular kernel," J. Austral. Math. Soc. Ser. B., v. 22, 1981, pp. 431-438.

26. R. DeVore \& K. SCherer, "Variable knot, variable degree spline approximation to $x^{\beta}$," in Quantitative Approximation (R. DeVore and K. Scherer, eds), Academic Press, New York, 1980, pp. 121-131. 